\title{
Regulatory role of the TLR4/JNK signaling pathway in sepsis-induced myocardial dysfunction
}

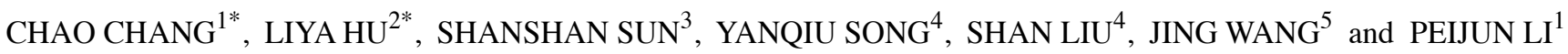 \\ ${ }^{1}$ Department of Cardiovascular Surgical ICU, Tianjin Chest Hospital, Nankai University, Tianjin 300222; \\ ${ }^{2}$ Department of Critical Care Medicine, The Third Central Hospital of Tianjin, Tianjin 300170; \\ ${ }^{3}$ Department of Emergency, Cangzhou Central Hospital, Cangzhou, Hebei 061001; ${ }^{4}$ Tianjin Cardiovascular Institute, \\ Tianjin Chest Hospital, Tianjin 300222; ${ }^{5}$ Department of Pathology, Tianjin Chest Hospital, \\ Nankai University, Tianjin 300222, P.R. China
}

Received June 10, 2020; Accepted October 30, 2020

DOI: $10.3892 / \mathrm{mmr} .2021 .11973$

\begin{abstract}
Sepsis is a life-threatening organ dysfunction caused by a dysregulated host response to infection, and is a leading cause of mortality worldwide. Myocardial dysfunction is associated with poor prognosis in patients with sepsis and contributes to a high risk of mortality. However, the pathophysiological mechanisms underlying sepsis-induced myocardial dysfunction are not completely understood. The aim of the present study was to investigate the role of toll-like receptor 4 (TLR4)/c-Jun N-terminal kinase (JNK) signaling in pro-inflammatory cytokine expression and cardiac dysfunction during lipopolysaccharide (LPS)-induced sepsis in mice. C57BL/6 mice were pretreated with TAK-242 or saline for $1 \mathrm{~h}$ and then subjected to LPS (12 mg/kg, intraperitoneal) treatment. Cardiac function was assessed using an echocardiogram. The morphological changes of the myocardium were examined by hematoxylin and eosin staining and transmission electron microscopy. The serum protein levels of cardiac troponin I (cTnI) and tumor necrosis factor- $\alpha$ (TNF- $\alpha)$ were determined by an enzyme-linked immunosorbent assay (ELISA). The TLR4 and JNK mRNA levels were analyzed via reverse transcription-quantitative PCR. TLR4, JNK and phosphorylated-JNK protein levels were measured by western blotting. In response to LPS, the activation of TLR4 and JNK in the myocardium was upregulated. There were significant increases in the serum levels of TNF- $\alpha$ and cTnI, as well as histopathological changes in the myocardium
\end{abstract}

Correspondence to: Professor Peijun Li, Department of Cardiovascular Surgical ICU, Tianjin Chest Hospital, Nankai University, 261 Taierzhuang South Road, Jinnan, Tianjin 300222, P.R. China

E-mail: peijunli-tch@outlook.com

*Contributed equally

Key words: sepsis, myocardial dysfunction, lipopolysaccharide, animal models, Toll-like receptor 4 , c-Jun N-terminal kinase signaling pathway and suppressed cardiac function, following LPS stimulation. Inhibition of TLR4 activation using TAK-242 led to a decrease in the activation of JNK and reduced the protein expression of TNF- $\alpha$ in plasma, and alleviated histological myocardial injury and improved cardiac function during sepsis in mice. The present data suggested that the TLR4/JNK signaling pathway played a critical role in regulating the production of pro-inflammatory cytokines and myocardial dysfunction induced by LPS.

\section{Introduction}

Sepsis, the most common cause of death among patients in the intensive care unit, is defined as life-threatening organ dysfunction caused by a dysregulated host response to infection (1). It is recognized as a global public health problem due to its high mortality and morbidity, as well as its substantial economic burden (2). Rudd et al (3) recently reported that an estimated 48.9 million cases of sepsis and 11.0 million sepsis-related deaths were recorded worldwide in 2017, representing $19.7 \%$ of all global deaths. Septic shock is a subset of sepsis that can lead to multi-organ dysfunction and rapid death (1). Myocardial dysfunction is recognized as a common complication of septic shock and contributes to adverse outcomes (4). Endotoxins, such as lipopolysaccharide (LPS), which is an important structural component of the gram-negative bacterial outer membrane, are important factors responsible for septic myocardial dysfunction (5). Previous studies have demonstrated that LPS can mimic the myocardial dysfunction of septic shock $(6,7)$. A number of underlying pathophysiological mechanisms, including genetic, molecular, metabolic and structural mechanisms, have been proposed to be involved in sepsis-induced myocardial dysfunction (8). However, the specific mechanisms have not yet been elucidated.

Toll-like receptors (TLRs) are the first line of defense in the mammalian immune system and recognize pathogen-associated molecular patterns, such as lipoproteins, LPS, flagellin and nucleic acids of bacterial origin (9). Among these receptors, TLR4 has been identified as the only receptor for LPS and plays a key role in LPS-mediated inflammatory responses and myocardial dysfunction (6). The intracellular signaling 
pathways activated after LPS-TLR4 binding have been classified as the myeloid differentiation primary response protein 88 (MyD88)-independent and TIR-containing adapter inducing interferon- $\beta$ (TRIF)-dependent pathway (10). The MyD88-independent pathway induces the activation of nuclear factor- $\kappa \mathrm{B}$ and mitogen-activated protein kinases (MAPKs), thus leading to the release of pro-inflammatory cytokines and regulation of cardiac function during sepsis (11). MAPKs, including p38, extracellular signal-regulated kinase (ERK)1/2, c-Jun N-terminal kinase (JNK) and ERK5 (12), transduce a variety of extracellular signals that regulate the cellular response implicated in proliferation, differentiation, apoptosis, stress and inflammatory response $(13,14)$ and play an important role in regulating sepsis-induced cardiac dysfunction (15). JNK, also known as stress-activated protein kinase, is a member of the MAPK family. However, the role of the JNK signaling pathway in tumor necrosis factor- $\alpha$ (TNF- $\alpha$ ) expression and myocardial dysfunction induced by sepsis has not yet been clearly defined. The majority of previous studies $(16,17)$ have reported that the activation of the JNK signaling pathway promotes the production of pro-inflammatory cytokines and the development of LPS-induced cardiac dysfunction. Research from Peng et al (18) revealed that LPS activates JNK1, thus leading to the inhibition of TNF- $\alpha$ expression and improvement of the myocardial function in sepsis. In addition, only a few studies have investigated whether TLR4 mediates LPS-induced myocardial dysfunction by modulating the JNK signaling pathway. Thus, the aim of the present study was to examine the changes in cardiac function, myocardial histopathology and TNF- $\alpha$ expression during LPS stimulation. Furthermore, this study also attempted to explore the regulatory role of the TLR4/JNK signaling pathway in LPS-induced TNF- $\alpha$ expression and myocardial dysfunction. The results demonstrated that the production of TNF- $\alpha$ increased significantly, cardiac function decreased, and the activities of TLR4 and JNK in the myocardium were upregulated during LPS stimulation. Whereas, inhibition of TLR4 activation downregulated JNK activation, reduced $\mathrm{TNF}-\alpha$ expression and improved cardiac function in response to LPS. The present results suggested that TLR4 mediated myocardial dysfunction by regulating the JNK signaling pathway during sepsis.

\section{Materials and methods}

Animals. A total of 54 male wild-type C57BL/6 mice (8-12 weeks old and weighing 22-28 g), were purchased from the Laboratory Animal Center, Academy of Military Medical Sciences, [certificate no. SCXK (Jing) 2014-0013; Beijing, China]. The mice were housed in a specific pathogen-free environment at a constant temperature $\left(22 \pm 1^{\circ} \mathrm{C}\right)$ with $50 \%$ humidity and 12-h light/dark cycles. All mice had free access to food and water. All the experimental procedures were approved by the Animal Experiments Ethics Committee of Nankai University (Tianjin, China; approval no. 10011).

Experimental protocol. To induce sepsis, mice were injected intraperitoneally (i.p.) with $12 \mathrm{mg} / \mathrm{kg}$ LPS (from Escherichia coli, a phenol extract of serotype 011:B4; Sigma-Aldrich; Merck $\mathrm{KGaA}$ ), a dose that was sufficient to induce cardiac dysfunction as determined in our preliminary experiment (19).
Mice were randomly assigned to three groups: Sham group $(n=18)$, LPS group $(n=18)$ and TAK-242 group $(n=18)$. Mice in the LPS and TAK-242 groups were i.p. treated with saline or a TLR4 inhibitor (TAK-242; $2 \mathrm{mg} / \mathrm{kg}$ ), respectively, followed by LPS $(12 \mathrm{mg} / \mathrm{kg}$, i.p.) $1 \mathrm{~h}$ later. Mice in the sham group were injected with equal $0.9 \%$ saline. For time course experiments, mice were sacrificed at 3, 12 and $24 \mathrm{~h}$ after LPS or saline injection ( $n=6$ for each time point). Left ventricular (LV) function was assessed at 3, 12 and $24 \mathrm{~h}$ after LPS injection during anesthesia. After measurement of LV function, mice were euthanized by excessive inhalation of isoflurane, and then the plasma and ventricular myocardium were collected and stored at $-80^{\circ} \mathrm{C}$.

Echocardiographic assessment of LV function. A Vevo 2100 ultrahigh resolution small animal ultra-sound imaging system (VisualSonics, Inc.) with a MS-400 ultrasound scanning transducer was used to evaluate LV function (20-22). Briefly, mice were anesthetized with $1-1.25 \%$ isoflurane via a mask, and their chest was shaved before the animals were placed in the supine position on a $37^{\circ} \mathrm{C}$ pad. Warm electrode gel was applied to the limb leads, allowing for the electrocardiogram and respiration rate to be recorded during ultrasound imaging. Briefly, an even layer of ultrasound coupling agent was spread on the mouse thoracic, and the console was used at a downward sloping angle of $30-45^{\circ}$. Two-dimensional cine loops and guided M-mode frames were recorded from the parasternal long axis. The following parameters were measured as indicators of function: LV end diastolic diameter (LVEDD), LV end systole diameter (LVESD), LV end diastolic volume (LVEDV), LV end systolic volume (LVESV), $\mathrm{LV}$ ejection fraction percentage (LVEF\%), and LV fractional shortening percentage (LVFS\%). All data were analyzed off-line at the end of the study with the software in-built in the ultrasound system (20).

Histopathological examination of myocardial tissues. Immediately after the sacrifice of the mice, their hearts were removed, fixed in a $10 \%$ paraformaldehyde solution for $24 \mathrm{~h}$ at room temperature and embedded in paraffin. Serial sections $(4 \mu \mathrm{m})$ of the ventricle were affixed to slides, deparaffinized, and stained with hematoxylin and eosin (H\&E) for $5 \mathrm{~min}$ at room temperature. Pathological changes of the myocardial tissues were observed under a light microscope (magnification, $\mathrm{x} 400)$.

Transmission electron microscopy. Transmission electron microscopy was performed as described in a previous study by Wang et al (23). After pretreatment, the samples were cut into thin sections and visualized at $120 \mathrm{kV}$ with a $\mathrm{H} 7650$ transmission electron microscope (Hitachi, Ltd.). A total of 10-15 micrographs per sample were obtained using a Philips CM12 (Philips Medical Systems, Inc.) by random sampling.

Measurement of cardiac troponin I (cTnI) and TNF- $\alpha$ protein levels. cTnI and TNF- $\alpha$ protein levels in the plasma were determined using a mouse cTnI ELISA kit (Biotopped Life Sciences; cat. no. TOPEL02104) and a TNF- $\alpha$ ELISA kit (BioLegend; cat. no. 430907) according to the manufacturer's instructions. 
Table I. Primers used for reverse transcription-quantitative PCR.

\begin{tabular}{|c|c|}
\hline Gene & Primer sequences $\left(5^{\prime} \rightarrow 3^{\prime}\right)$ \\
\hline \multirow[t]{2}{*}{ TLR4 } & F: GCTAAGTGCCGAGTCTGAGTGTAA \\
\hline & R TGCAGCCTTTCAGAAACACATT \\
\hline \multirow[t]{2}{*}{ JNK } & F: GCTCTCAGCATCCATCGTCTTC \\
\hline & R: AGGTCCAGCTGATGCTTCTAGACT \\
\hline \multirow[t]{2}{*}{ GAPDH } & F: СТCTGCTCCTCCCTGTTCCA \\
\hline & R: ATACGGCCAAATCCGTTCAC \\
\hline
\end{tabular}

F, forward; R, reverse; TLR4, toll-like receptor 4; JNK, c-Jun N-terminal kinase.

Reverse transcription-quantitative PCR (RT-qPCR). Total RNA was extracted from the myocardial tissues using TRIzol ${ }^{\circledR}$ reagent (Ambion; Thermo Fisher Scientific, Inc.), following the manufacturer's instructions. RT of the purified RNA $(4 \mu \mathrm{g})$ was performed using random primers and the RevertAid First Strand cDNA Synthesis kit (Thermo Fisher Scientific, Inc.), according to the manufacturer's instructions. TB Green Premix EX Taq II (Tli RNase H Pus) (Takara Bio, Inc.; cat. no. RR820A) in a CFX96 system (Bio-Rad Laboratories, Inc.) was used to determine the mRNA expression of TLR4 and JNK. All reactions were performed in triplicate. The sequences of the sense and antisense primers used for amplification are listed in Table I. The relative expression of the target genes was determined by calculating the values of the $\Delta$ cycle quantification $(\Delta \mathrm{Cq})$ by normalizing the average $\mathrm{Cq}$ value to that of the endogenous control (GAPDH), and then calculating the $2^{-\Delta \Delta \mathrm{Cq}}$ values (24).

Western blot analysis. The Whole Protein Extraction kit (Applygen Technologies, Inc.) was used to extract the whole protein of myocardial tissue, according to manufacturer's instructions. The concentration of whole protein was determined with a Qubit ${ }^{\mathrm{TM}}$ Protein Assay kit (Thermo Fisher Scientific, Inc.), according to the manufacturer's instructions, and the mean values of concentration were determined. The samples were stored at $-80^{\circ} \mathrm{C}$ until use. Following quantification, equal amounts of protein samples $(11.25 \mathrm{mg})$ from the homogenized total tissues were separated using 5\% stacking and $10 \%$ separating gels, and subsequently transferred to a PVDF membrane. After blocking at room temperature for $2 \mathrm{~h}$ with $5 \%$ non-fat milk and Tris-buffered saline with $0.05 \%$ Tween-20 (TBST) or 5\% bovine serum albumin (cat. no. A8010; Beijing Solarbio Science \& Technology Co., Ltd.) and washing with TBST, the membranes were incubated overnight at $4^{\circ} \mathrm{C}$ with primary antibodies against TLR4 (1:100; Santa Cruz Biotechnology, Inc.; cat. no. sc-293072), JNK (1:500; Abcam; cat. no. ab112501), phosphorylated (p)-JNK (1:500; Abcam; cat. no. ab4821) or GAPDH (1:10,000; ProteinTech Group, Inc.; cat. no. 60004-1-lg). Subsequently, the membranes were rinsed and incubated with the corresponding secondary antibody, either horseradish peroxidase-conjugated goat anti-rabbit or goat anti-mouse IgG (1:5,000; Boster Biological Technology; cat. nos. BA1055 and BA1051), for $2 \mathrm{~h}$ at room temperature. Immunoblots were visualized with an enhanced chemiluminescence reagent (Bio-Rad Laboratories, Inc.), and developed and analyzed using Quantity One software (version 4.6; Bio-Rad Laboratories, Inc.). The protein signals were quantified by gray scale values. GAPDH was used as an internal control. The total protein level was normalized to the GAPDH protein level, and the level of p-JNK to total JNK were presented.

Statistical analysis. All experiments were repeated $\geq 3$ times. All data are presented as the mean \pm SD. Statistical analysis was performed using SPSS 22.0 statistical software (IBM Corp.) and graphs were generated using Prism 6.0 software (GraphPad Software, Inc.). Comparisons among multiple groups were analyzed using one-way ANOVA followed by the SNK post hoc test. $\mathrm{P}<0.05$ was considered to indicate a statistically significant difference.

\section{Results}

Changes in cardiac function. The beneficial effect of TAK-242 pretreatment on LPS-induced cardiac dysfunction was confirmed by quantitative analysis of echocardiograms. Representative echocardiograms in the different groups are presented in Fig. 1. The results of echocardiography analysis in various groups are summarized in Fig. 2. Compared with the values in the sham groups, the LPS groups showed a significant decrease in LVEDD at $24 \mathrm{~h}$, and in LVEDV at 12 and $24 \mathrm{~h}(\mathrm{P}<0.05)$, as well as a significant increase in LVESD and LVESV at 3 and $12 \mathrm{~h}(\mathrm{P}<0.05)$, and a significant decrease in LVEF\% and LVFS\% at all time points after LPS injection $(\mathrm{P}<0.05)$. However, compared with the values in the LPS groups, pretreatment with TAK-242 induced a significant increase in LVEDD and LVEDV at 12 and $24 \mathrm{~h}(\mathrm{P}<0.05)$, alongside a significant reduction in LVESD and LVESV at $3 \mathrm{~h}$ $(\mathrm{P}<0.05)$, and a significant increase in LVEF\% and LVFS\% at $3 \mathrm{~h}$ following LPS injection $(\mathrm{P}<0.05)$.

Histological changes in the myocardium. To directly observe the effect of TAK-242 pretreatment on the LPS-induced alterations in myocardial structure, the histology of the LV myocardial tissues was examined (Fig. 3). H\&E staining showed that distinct myocardial injury occurred in the myocardial tissues of the LPS groups, including myocardial interstitial edema, prominent hemorrhaging, rupture of myocardial fibers, myocardial cell swelling, degeneration, loss of transverse striations and infiltration of leukocytes. Under the transmission electron microscope, the myocardial ultrastructure in the LPS groups was damaged, which was characterized by myofibrillar disarray, cellular disorganization, disarrangement of sarcomere and/or disruption, mitochondrial swelling and cracking, disappearance of mitochondrial crista, as well as autophagic vacuoles. As time increased, the degree of myocardial damage in the LPS groups gradually worsened. However, the LPS-induced myocardial injuries were ameliorated by pretreatment with TAK-242.

Serum levels of cTnI and TNF- $\alpha$. Significant increases in the enzyme cTnI, a myocardial injury marker, were observed in the LPS groups compared with the values of the sham groups, and the level of cTnI increased gradually with time $(\mathrm{P}<0.05)$. 
$3 \mathrm{~h}$
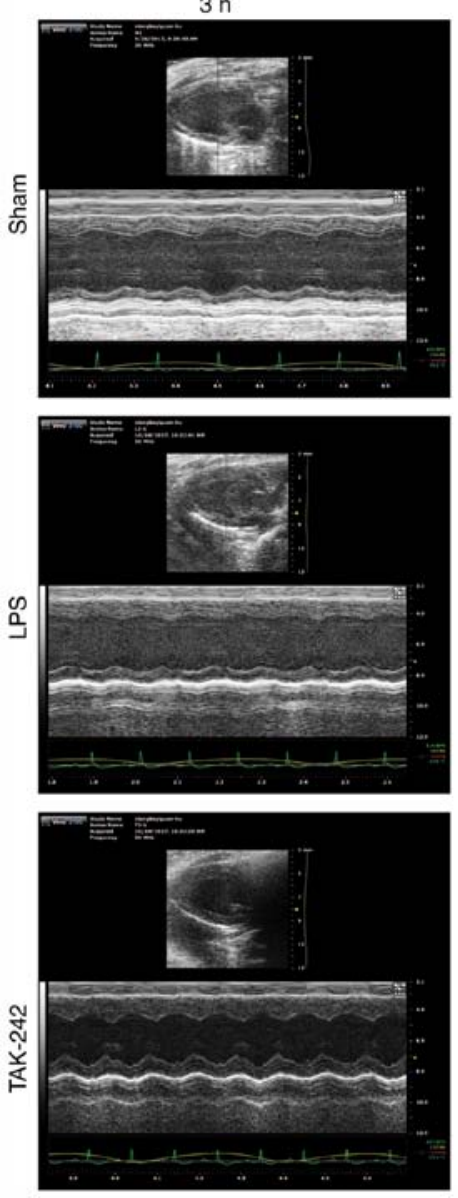

$12 \mathrm{~h}$
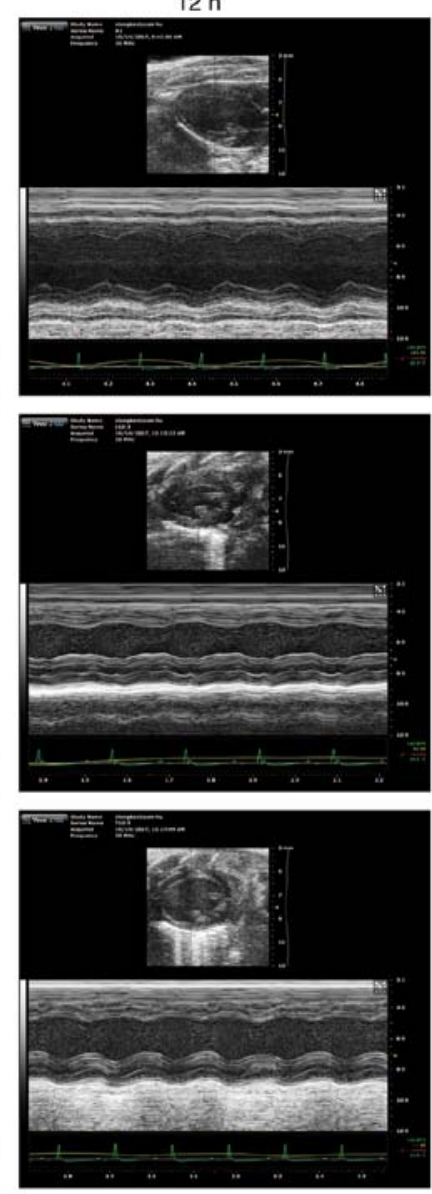

$24 \mathrm{~h}$
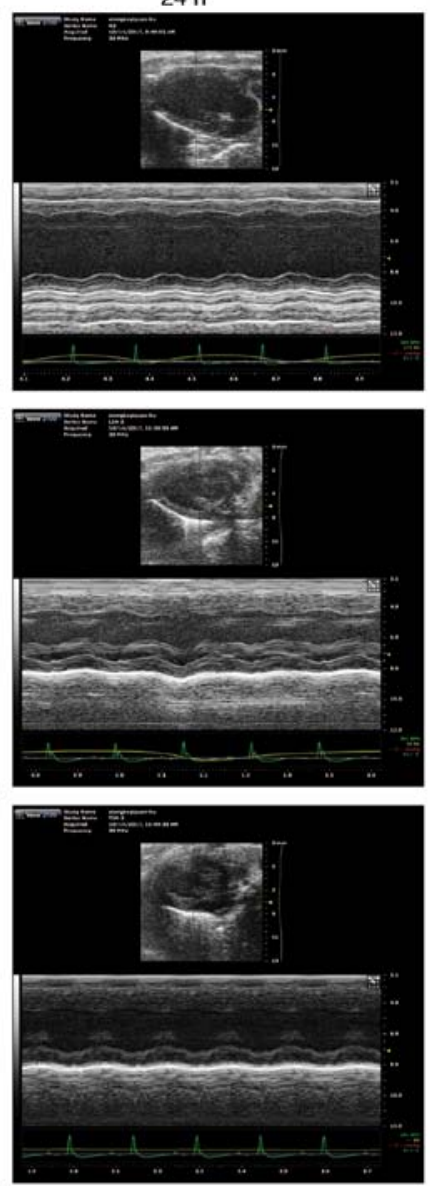

Figure 1. Representative echocardiographic images (M-mode) in different groups. Left, representative echocardiographic images in sham group (top), LPS group (middle) and TAK-242 group (bottom) at $3 \mathrm{~h}$ after LPS or saline injection. Middle, representative echocardiographic images in sham group (top), LPS group (middle) and TAK-242 group (bottom) at $12 \mathrm{~h}$ after LPS or saline injection. Right, representative echocardiographic images in sham group (top), LPS group (middle) and TAK-242 group (bottom) at $24 \mathrm{~h}$ after LPS or saline injection. LPS, lipopolysaccharide.

Pretreatment with TAK-242 significantly reduced the level of cTnI compared with that of the LPS group $(\mathrm{P}<0.05)$. Compared with the levels of the sham groups, the serum TNF- $\alpha$ level increased significantly in the LPS groups $(\mathrm{P}<0.05)$ and reached the maximal level at $3 \mathrm{~h}$ following LPS injection. Pretreatment with TAK-242 induced a significant decrease in the serum TNF- $\alpha$ level $(\mathrm{P}<0.05)$, compared with that of the LPS groups (Fig. 4).

TLR4 and JNK gene expression in the myocardium. As shown in Fig. 5, the relative TLR4 and JNK mRNA expression levels were significantly increased in response to LPS $(\mathrm{P}<0.05)$ and reached the maximal levels at 3 and $12 \mathrm{~h}$ after LPS injection, respectively. Compared with that of the LPS groups, pretreatment with TAK-242 significantly downregulated the expression levels of TLR4 and JNK mRNA at 3 and $12 \mathrm{~h}$ after LPS injection, respectively $(\mathrm{P}<0.05)$.

TLR4, JNK and p-JNK protein expression in the myocardium. As revealed by western blot analysis (Fig. 6), compared with that of the sham groups, the relative protein expression of TLR4 and p-JNK was significantly increased in response to LPS $(\mathrm{P}<0.05)$, and reached the peak $\sim 3$ and $12 \mathrm{~h}$ following LPS injection, respectively. Pretreatment with TAK-242 significantly reduced the protein levels of TLR4 and p-JNK
$(\mathrm{P}<0.05)$. LPS induced a significant increase in the protein level of the total JNK, compared with the level of the sham groups $(\mathrm{P}<0.05)$. Although TAK-242 significantly decreased the protein expression of total JNK compared with the LPS groups at 3 and $12 \mathrm{~h}(\mathrm{P}<0.05)$, there was no significant difference at $24 \mathrm{~h}(\mathrm{P}>0.05)$.

\section{Discussion}

The present study demonstrated that, in response to LPS, the activation of TLR4 and JNK in the myocardium was upregulated, and the serum levels of TNF- $\alpha$ and $c T n I$ were increased. Pathological myocardial damage was observed using H\&E staining and transmission electron microscopy, and cardiac function was shown to be reduced. Inhibition of TLR4 activation led to the reduction of JNK activation and protein expression of TNF- $\alpha$ in the plasma, and alleviated histopathological myocardial injury and improved cardiac function during sepsis in mice. The present data supported the notion that the TLR4/JNK signaling pathway plays an important role in myocardial dysfunction induced by sepsis.

Sepsis is a complicated syndrome that begins with a systemic immune response to an infection and can progress to septic shock leading to multiple organ failure and death (25). Although the identification of clinical biomarkers in patients 
A

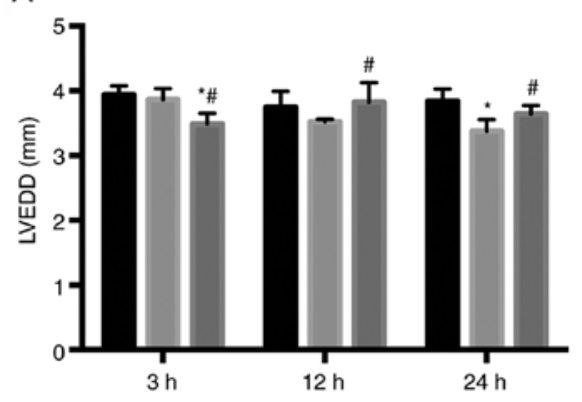

C

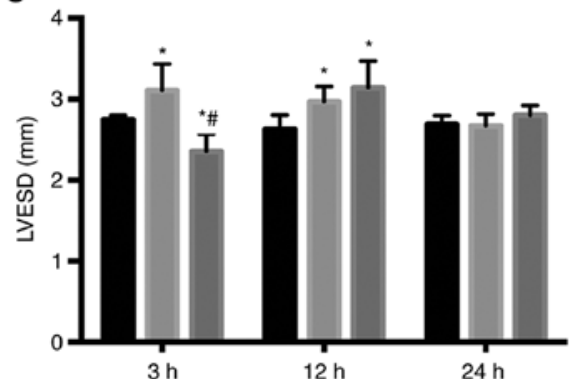

E

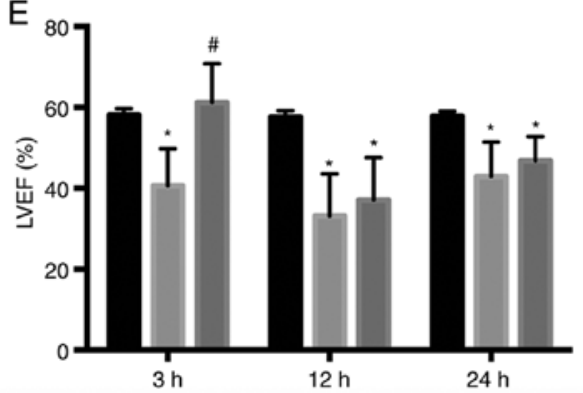

B
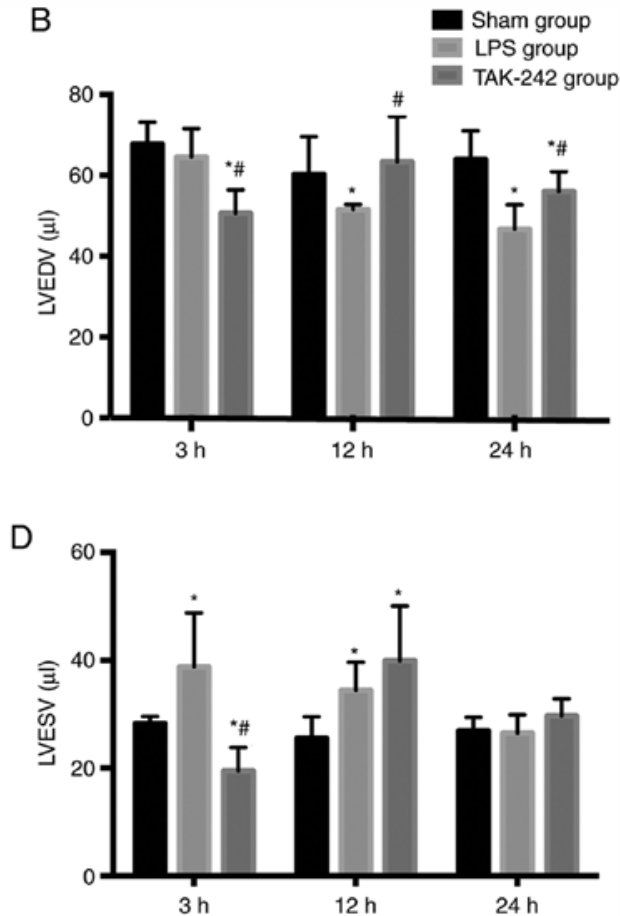

F

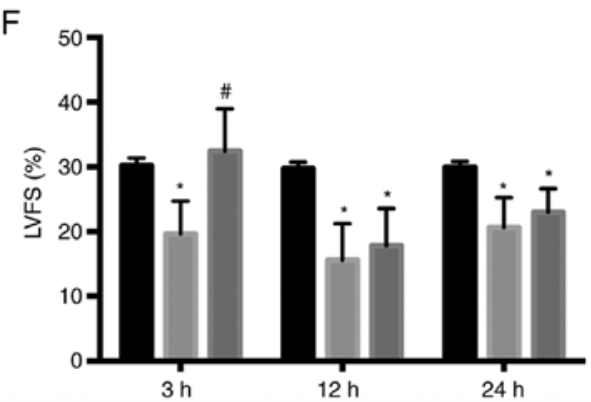

Figure 2. Echocardiographic characterization of cardiac function in different groups. Quantitative assessment of dilation and systolic function of heart based on (A) LVEDD, (B) LVEDV, (C) LVESD, (D) LVESV, (E) LVEF and (F) LVFS. Data are expressed as the mean \pm SD from six animals in each group. ${ }^{*} \mathrm{P}<0.05$ vs. sham group; ${ }^{\#} \mathrm{P}<0.05$ vs. LPS group. LVEDD, left ventricular end diastolic diameter; LVESD, left ventricular end systole diameter; LVEDV, left ventricular end diastolic volume; LVESV, left ventricular end systolic volume; LVEF, left ventricle ejection fraction; LVFS, left ventricle fractional shortening; LPS, lipopolysaccharide.

to facilitate earlier diagnosis and rapid therapeutic intervention is likely to have the greatest impact on improving prognosis, understanding the underlying mechanisms of the disease and evaluating the therapeutic efficacy of novel interventions using animal models are also of critical importance. Animal models of sepsis are broadly divided into three categories: Toxemia models, bacterial infection models and host-barrier disruption models (26). The advantage of toxemia models is that there is a rapid onset of pathological changes, and they can have relatively low inter-animal variability because the exact dose and route of administration can be standardized (26). In addition, toxemia models are often used to study the basic biology of septic shock, and in particular, they are employed in mechanistic studies on the role of TLR signaling (26). Considering the aforementioned reasons, in the present study, the sepsis models were established by i.p. injection of $12 \mathrm{mg} / \mathrm{kg} \mathrm{LPS}$, a dose demonstrated to ensure cardiac dysfunction in our preliminary experiment (19).

TLR4 is a type I transmembrane protein and has a modular structure composed of an extracellular domain formed by 17-31 leucine-rich repeats and an intracellular domain that is known as the toll-IL-1 receptor domain, which is responsible for signaling transmission (27). Since the identification of TLR4 as the LPS receptor, it has been assumed that this molecule triggers all the responses to LPS (28). The TLR4 signaling pathway plays an important role in initiating the innate immune response and inflammation in sepsis. Typically, activation of TLR4 is preceded by binding of LPS to CD14 protein, and then CD14 transfers the LPS to the TLR4/myeloid differentiation factor 2 (MD-2) complex, which dimerizes and triggers MyD88- and TRIF-dependent production of pro-inflammatory cytokines (29). The signaling pathway associated with MAPKs is an important signal transduction pathway that mediates sepsis-induced myocardial dysfunction. It has been reported that the activation of ERK, JNK and p38 is increased in response to LPS stimulation in cardiomyocytes (30), which is consistent with the results of the current study that found LPS administration increased the phosphorylation levels of JNK. A previous study demonstrated that TLR4/MyD88 triggers a signaling cascade that leads to 

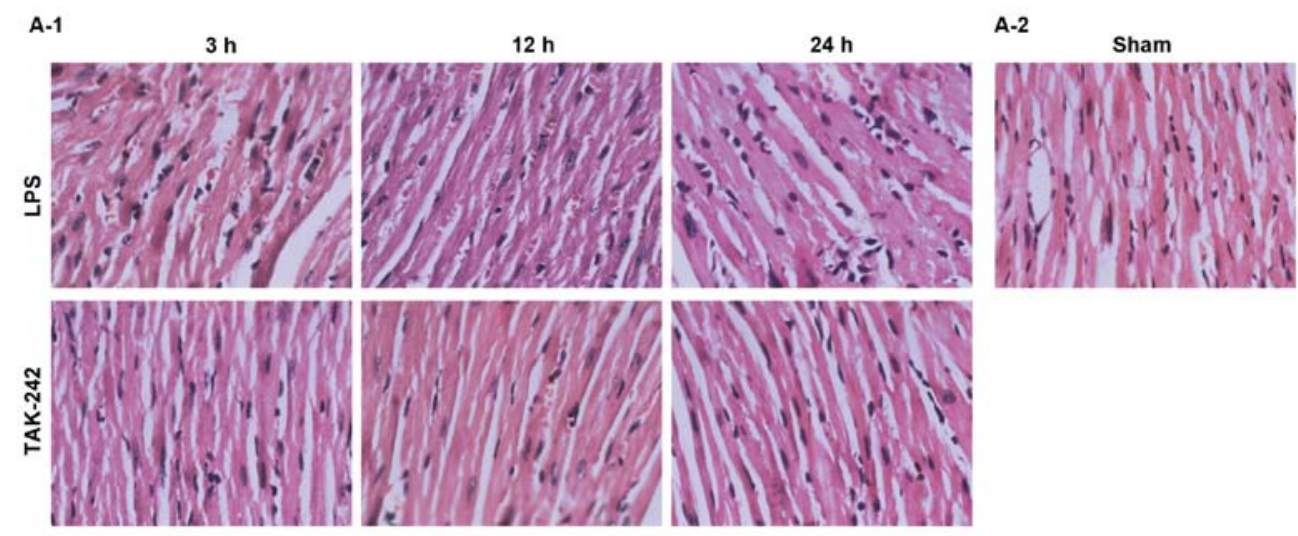

B-1

$3 \mathrm{~h}$

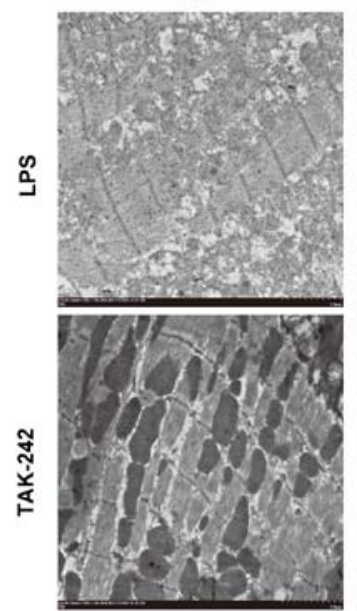

$12 \mathrm{~h}$

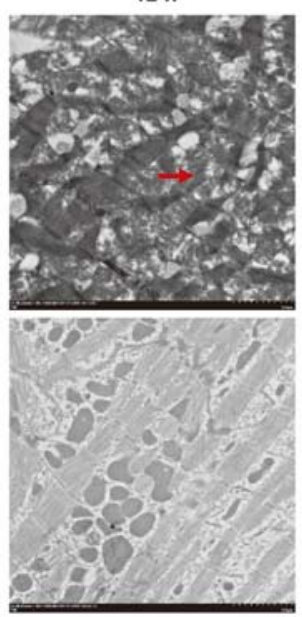

$24 \mathrm{~h}$

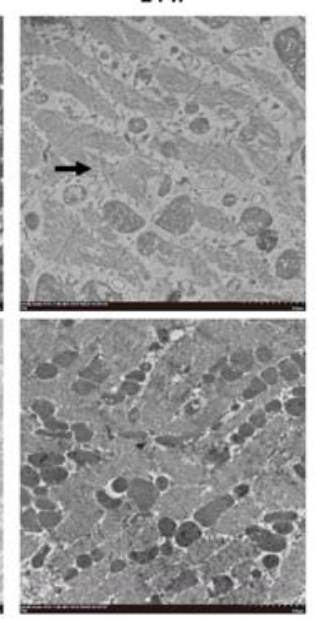

B-2

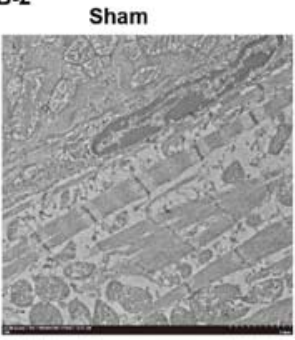

Figure 3. Histological changes of myocardium in different groups. (A) Representative images of hematoxylin and eosin-stained sections of the mouse heart tissues in different groups. (A-1) The LPS (top) and TAK-242 (bottom) groups. (A-2) The sham group. Magnification, x400. (B) Representative images of transmission electron micrographs of the mouse heart in different groups. Black arrows indicate myofibrillar disarray, and red arrows indicate mitochondrial swelling and cracking. (B-1) The LPS (top) and TAK-242 (bottom) groups. (B-2) The sham group. Magnification, x3,000. LPS, lipopolysaccharide.

A

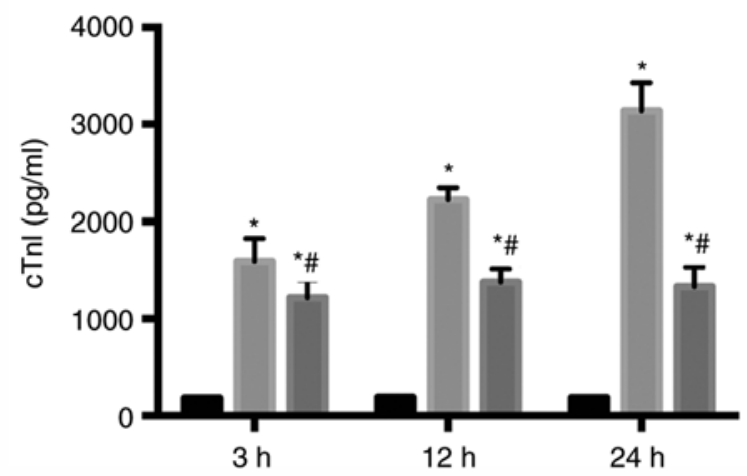

$\mathrm{B}$

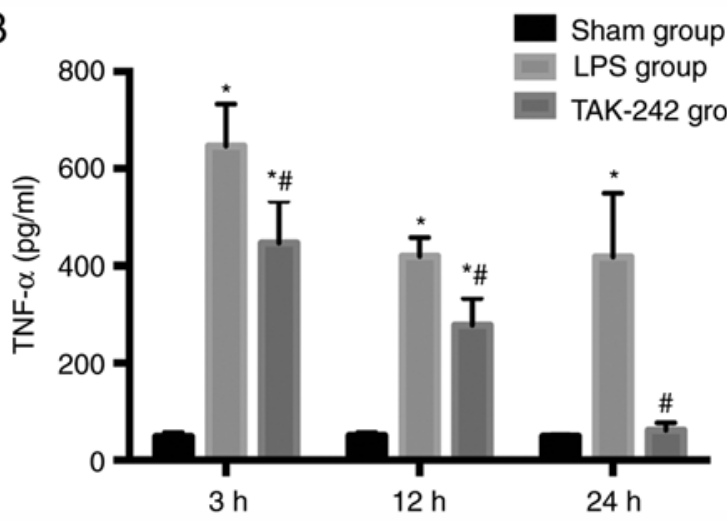

Figure 4. Protein levels of cTnI and TNF- $\alpha$ of mouse serum in different groups. Statistical results from ELISA for (A) cTnI and (B) TNF- $\alpha$. Data are expressed as the mean \pm SD from six animals in each group. ${ }^{*} \mathrm{P}<0.05$ vs. sham group; ${ }^{\prime \prime} \mathrm{P}<0.05$ vs. LPS group. cTnI, cardiac troponin I; TNF- $\alpha$, tumor necrosis factor- $\alpha$; ELISA, enzyme linked immunosorbent assay; LPS, lipopolysaccharide.

early-phase activation of MAPKs (29). In the present study, to investigate whether TLR4 plays a role in the activation of JNK in response to LPS, TAK-242 was employed to block TLR4 signaling. TAK-242 is a small-molecule compound that selectively inhibits the TLR4 signaling pathway by binding directly to the intracellular domain of TLR4 (27). Pretreatment with TAK-242 inhibited the activation of JNK induced by LPS, indicating that JNK is located downstream of TLR4 signaling.

Myocardial dysfunction is regarded as a well-recognized clinical manifestation of sepsis and septic shock, and is characterized by biventricular dilation, reduced ejection fraction (EF), and a recovery period of 7-10 days in patients with sepsis (31). Echocardiographic techniques have revealed that 


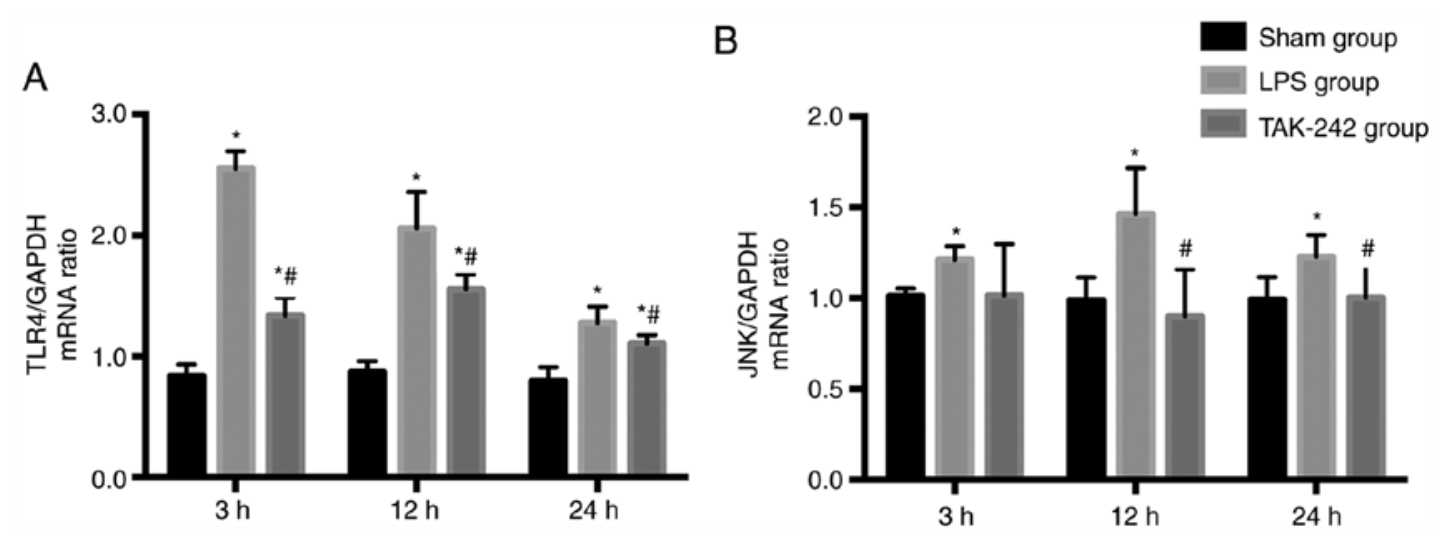

Figure 5. TLR4 and JNK gene expression in mouse hearts from different groups. The relative expression levels of cardiac (A) TLR4 and (B) JNK mRNA were assessed by reverse transcription-quantitative PCR. Results were normalized to GAPDH. Data are expressed as mean $\pm \mathrm{SD}\left(\right.$ each group, $\mathrm{n}=6$ ). ${ }^{*} \mathrm{P}<0.05 \mathrm{vs}$. sham group; " $\mathrm{P}<0.05$ vs. LPS group. TLR4, toll-like receptor 4; JNK, c-Jun N-terminal kinase; LPS, lipopolysaccharide.

A-1

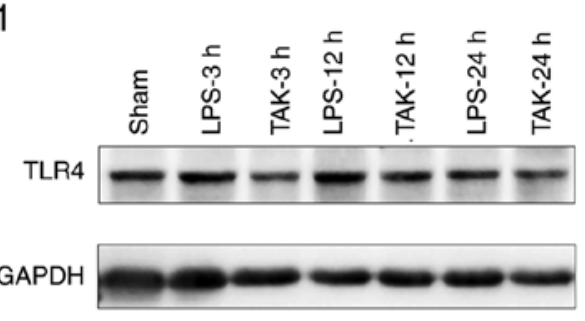

B-1
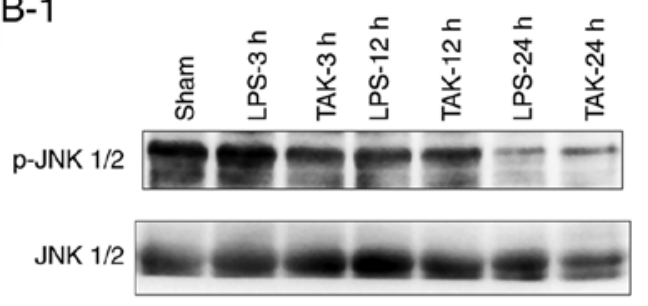

GAPDH

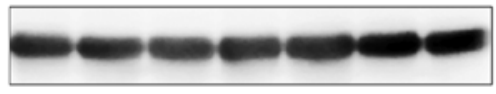

A-2

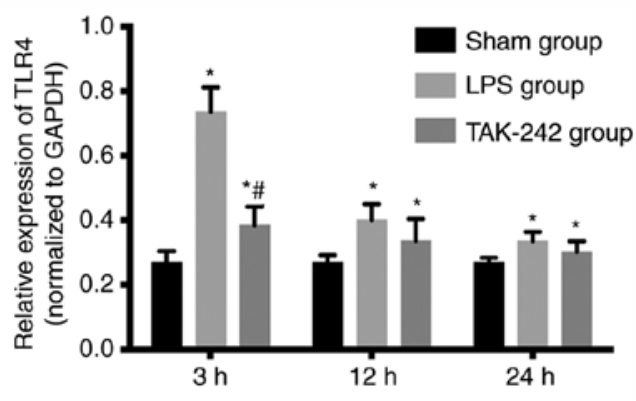

B-2

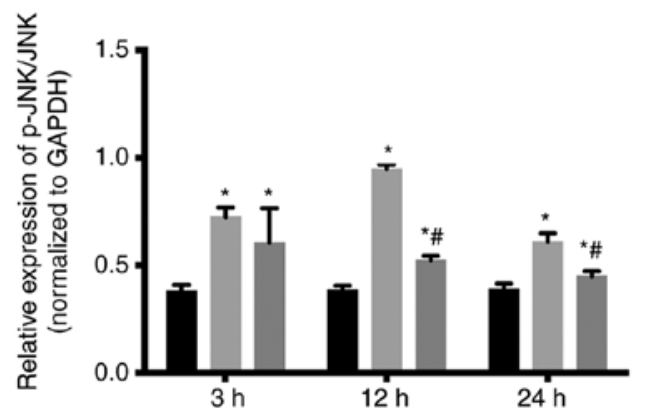

B-3

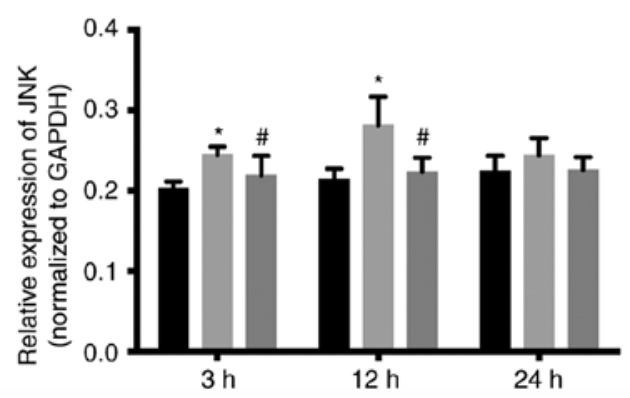

Figure 6. TLR4, JNK and p-JNK protein expression in the mouse hearts from different groups. Representative western blotting images showing protein expression of (A-1) TLR4 and (B-1) total and p-JNK in the mouse heart from various groups. Semi-quantification of western blot analysis, (A-2) TLR4, (B-2) p-JNK/JNK and (B-3) expression. Protein expression shown as relative densitometric absorption units (left axis). Data are expressed as the mean \pm SD (each group, $n=6$ ). ${ }^{*} \mathrm{P}<0.05$ vs. sham group; ${ }^{*} \mathrm{P}<0.05$ vs. LPS groups. TLR4, toll-like receptor 4; JNK, c-Jun N-terminal kinase; LPS, lipopolysaccharide; p-, phosphorylated.

either systolic or diastolic dysfunction is commonly present in sepsis (32). Currently, two-dimensional echocardiography is the most common diagnostic tool for assessing myocardial dysfunction in sepsis (33), and the M-mode-derived fractional shortening, EF and ventricular dimensions are regarded as common parameters to evaluate cardiac function (20). Among these parameters, $\mathrm{EF}$ is most commonly employed to evaluate LV systolic function (34). Diastolic dysfunction occurs in $\sim 50 \%$ 
patients with sepsis and is associated with high mortality (35). LV dilation can be assessed by estimating the LVEDV, a simple measure of ventricular dilation that is associated with worse clinical outcomes (34). In the present study, suppressed cardiac function was confirmed by reduced EF\% and $\mathrm{FS} \%$ in response to LPS, and was improved by pretreatment with TAK-242 at $3 \mathrm{~h}$ following LPS injection, which suggested that TLR4/JNK signaling plays an important role in LPS-induced systolic dysfunction. However, the present study showed that TAK-242 failed to reverse the inhibitory effect of LPS on LVEF\% and LVFS\% at 12 and $24 \mathrm{~h}$. The reasons for this finding may be because TAK-242 has been demonstrated to act early in the process of TLR4 signaling (36) or it may be related to the half-life of TAK-242. Further studies will explore whether TAK-242 can reverse the inhibitory effect of LPS on LVEF\% and LVFS\% at different time points through continuous and intermittent administration of TAK-242. Contrary to previous studies (37), the current data showed that the decreases in LVEDD and LVEDV were induced by LPS stimulation, which may be related to decompensation of ventricular diastolic function and further studies are necessary to explore the reasons.

However, there were some limitations of the present study. The results showed that LPS had no significant effect on LVEDD, but in the TAK-242 group, there was either a decrease or an increase in LVEDD at 3 and $12 \mathrm{~h}$, respectively. These results suggested that the effect of TAK-242 was independent of LPS exposure. TLR4 is an important pathway and is most commonly known for inducing inflammation in response to LPS, which is the most understood TLR4 agonist. However, it has also been established that TLR4, as a single protein, has a remarkably low affinity for LPS. Triggering the TLR4 pathway by LPS requires at least four co-receptors: MD-2, CD14 and CD11b bound to CD18 (38). In response to LPS, TLR4-expressing normal cells significantly increase the production of inflammatory factors due to the combined upregulation of TLR4, its intracellular adapters and co-receptors, which enhances cooperation among the pathway's components (38). TAK-242 is a small-molecule compound that selectively inhibits the TLR4 signaling pathway by binding directly to Cys747 in the intracellular TLR4 domain (27). Therefore, theoretically, the effect of TAK-242 cannot be independent of LPS exposure. In order to examine the effect of TAK-242 on various parameters, an additional group of sham animals treated with TAK-242 is needed, so further relevant experiments will be performed in the future.

Previous evidence suggests that structural abnormalities may play a critical role in the pathophysiology of sepsis-induced cardiac dysfunction (39). In previous years, cardiac biomarkers have been used to detect myocardial injury (40). Troponin-I, a sensitive and specific marker of myocardial injury, is the subunit of the troponin complex. Previous studies have demonstrated that cardiac troponin release in sepsis is related to LV dysfunction and poor outcomes $(41,42)$. In the present study, consistent with previous findings $(40,43)$, LPS-induced myocardial injury was confirmed by the loss of integrity of myocardial membranes on histological examination (Figs. 3 and 4) and elevation of the cTnI enzyme. Furthermore, myocardial injury and elevation of cTnI could be attenuated by TAK-242 administration. According to the current results, it could be suggested that the TLR4/JNK signaling pathway plays a critical role in myocardial injury induced by LPS.
Systemic inflammatory response syndrome (SIRS) is a key characteristic in the development of organ injury during sepsis and overactivated inflammatory response plays a critical role in sepsis (40). Although there is no definitive cause-and-effect association between systemic cytokine levels and survival outcomes in sepsis, numerous studies have reported that inhibition of the cardiac inflammatory processes is beneficial in sepsis-induced cardiac dysfunction $(43,44)$. The production of pro-inflammatory cytokines stimulated by LPS has been demonstrated to be one of the primary underlying mechanisms of cardiac dysfunction (44). As a trigger of inflammation, TNF- $\alpha$ participates in the production of IL- $1 \beta$, and together with IL-1 $\beta$, it induces the formation of secondary inflammatory factors, such as IL-6, resulting in an inflammatory cascade (45). In addition, cytokines, in particular TNF- $\alpha$, can also induce the release of additional inflammatory factors, such as inducible nitric oxide synthase (iNOS), cyclooxygenase-2 (COX-2) and reactive oxygen species (ROS), which eventually induce myocardial dysfunction $(46,47)$. Administration of TNF- $\alpha$ directly suppresses myocardial function in animal and human cardiomyocytes $(48,49)$, and treatment with anti-TNF- $\alpha$ protects cardiac function in sepsis animal models and patients with sepsis $(50,51)$. In the present study, LPS increased the production of TNF- $\alpha$ in mouse serum, which is supported by a previous finding that inflammatory cytokines such as TNF- $\alpha$ were induced by LPS in mice (30). Inhibition of TLR4 by pretreatment with TAK-242 significantly reduced the protein levels of TNF- $\alpha$ induced by LPS. These results indicated that TLR4/JNK signaling was an important pathway leading to TNF- $\alpha$ expression in response to LPS stimulation. However, the present study failed to include assays that demonstrated the differential expression of inflammatory-related proteins, such as iNOS, COX-2 and IL-6, and accumulation of ROS. Relevant experiments to determine the expression of inflammatory-related proteins will be performed in the future.

In conclusion, the present study demonstrated that the TLR4/JNK signaling pathway appeared to be of critical importance in sepsis, as its inhibition attenuated cardiac dysfunction and the overactivated inflammatory response. Therefore, this study indicated that the TLR4/JNK signaling pathway plays an important role in regulating myocardial dysfunction in sepsis.

\section{Acknowledgements}

Not applicable.

\section{Funding}

This study was supported by The Health and Family Planning Commission of Tianjin of China (grant no. 2014KY32).

\section{Availability of data and materials}

The datasets used and/or analyzed during the current study are available from the corresponding author on reasonable request.

\section{Authors' contributions}

PL and CC conceived, designed and coordinated the present study. LH performed the majority of experiments, analyzed 
the data and drafted the manuscript. SS, YS, SL and JW helped peform the experiments. All authors reviewed the results, and read and approved the final manuscript.

\section{Ethics approval and consent to participate}

All the experimental procedures were approved by the Animal Experiments Ethics Committee of Nankai University (Tianjin, China; approval no. 10011).

\section{Patient consent for publication}

Not applicable.

\section{Competing interests}

The authors declare that they have no competing interests.

\section{References}

1. Singer M, Deutschman CS, Seymour CW, Shankar-Hari M, Annane D, Bauer M, Bellomo R, Bernard GR, Chiche JD, Coopersmith CM, et al: The third international consensus definitions for sepsis and septic shock (Sepsis-3). JAMA 315: 801-810, 2016.

2. Markwart R, Saito H, Harder T, Tomczyk S, Cassini A, Fleischmann-Struzek C, Reichert F, Eckmanns T and Allegranzi B: Epidemiology and burden of sepsis acquired in hospitals and intensive care units: A systematic review and meta-analysis. Intensive Care Med 46: 1536-1551, 2020.

3. Rudd KE, Johnson SC, Agesa KM, Shackelford KA, Tsoi D, Kievlan DR, Colombara DV, Ikuta KS, Kissoon N, Finfer S, et al: Global, regional, and national sepsis incidence and mortality, 1990-2017: Analysis for the Global Burden of Disease Study. Lancet 395: 200-211, 2020.

4. Antonucci E, Fiaccadori E, Donadello K, Taccone FS, Franchi F and Scolletta S: Myocardial depression in sepsis: From pathogenesis to clinical manifestations and treatment. J Crit Care 29: 500-511, 2014

5. Buras JA, Holzmann B and Sitkovsky M: Animal models of sepsis: Setting the stage. Nat Rev Drug Discov 4: 854-865, 2005.

6. Fallach R, Shainberg A, Avlas O, Fainblut M, Chepurko Y, Porat E and Hochhauser E: Cardiomyocyte Toll-like receptor 4 is involved in heart dysfunction following septic shock or myocardial ischemia. J Mol Cell Cardiol 48: 1236-1244, 2010.

7. Suffredini AF, Fromm RE, Parker MM, Brenner M, Kovacs JA, Wesley RA and Parrillo JE: The cardiovascular response of normal humans to the administration of endotoxin. N Engl J Med 321: 280-287, 1989.

8. Fenton KE and Parker MM: Cardiac function and dysfunction in sepsis. Clin Chest Med 37: 289-298, 2016.

9. Gay NJ, Symmons MF, Gangloff M and Bryant CE: Assembly and localization of Toll-like receptor signalling complexes. Nat Rev Immunol 14: 546-558, 2014.

10. Conejeros I, Gibson AJ, Werling D, Muñoz-Caro T, Hermosilla C, Taubert A and Burgos RA: Effect of the synthetic Toll-like receptor ligands LPS, Pam3CSK4, HKLM and FSL-1 in the function of bovine polymorphonuclear neutrophils. Dev Comp Immunol 52: 215-225, 2015.

11. Song Y, Liu X, Yue H, Ji J, Dou H and Hou Y: Anti-inflammatory effects of benzenediamine derivate FC-98 on sepsis injury in mice via suppression of JNK, NF- $\mathrm{BB}$ and IRF3 signaling pathways. Mol Immunol 67: 183-192, 2015.

12. Chang C, Zhang C, Zhao X, Kuang X, Tang H and Xiao X Differential regulation of mitogen-activated protein kinase signaling pathways in human with different types of mitral valvular disease. J Surg Res 181: 49-59, 2013.

13. Sabio G and Davis RJ: TNF and MAP kinase signalling pathways. Semin Immunol 26: 237-245, 2014.

14. Plotnikov A, Zehorai E, Procaccia S and Seger R: The MAPK cascades: Signaling components, nuclear roles and mechanisms of nuclear translocation. Biochim Biophys Acta 1813: 1619-1633, 2011.
15. Feng F, Qi Y, Dong $C$ and Yang C: PVT1 regulates inflammation and cardiac function via the MAPK/NF- $\mathrm{kB}$ pathway in a sepsis model. Exp Ther Med 16: 4471-4478, 2018

16. Lee SM, Suk K and Lee WH: Myristoylated alanine-rich C kinase substrate (MARCKS) regulates the expression of proinflammatory cytokines in macrophages through activation of p38/JNK MAPK and NF- $\kappa$ B. Cell Immunol 296: 115-121, 2015.

17. Wang Z, Wu Q, Nie X, Guo J and Yang C: Infusion of esmolol attenuates lipopolysaccharide-induced myocardial dysfunction. J Surg Res 200: 283-289, 2016.

18. Peng T, Zhang T, Lu X and Feng Q: JNK1/c-fos inhibits cardiomyocyte TNF-alpha expression via a negative crosstalk with ERK and p38 MAPK in endotoxaemia. Cardiovasc Res 81: 733-741, 2009.

19. Hu L, Li P, Chang C, Liu S, Song Y,Zhao F and Liu T: Establishment and evaluation of mouse models of septic myocardial injury. Zhonghua Wei Zhong Bing Ji Jiu Yi Xue 30: 342-345, 2018 (In Chinese).

20. Chen J, Wei J, Wang L, Zhu Y, Li L, Akinyi M, Gao X and Fan G: Cardioprotection against ischemia/reperfusion injury by QiShenYiQi Pill ${ }^{\circledR}$ via ameliorate of multiple mitochondrial dysfunctions. Drug Des Devel Ther 9: 3051-3066, 2015.

21. Täng MS, Redfors B, Shao Y and Omerovic E: Velocity vector imaging fails to quantify regional myocardial dysfunction in a mouse model of isoprenaline-induced cardiotoxicity. Echocardiography 29: 818-826, 2012.

22. Teng B, Tilley SL, Ledent C and Mustafa SJ: In vivo assessment of coronary flow and cardiac function after bolus adenosine injection in adenosine receptor knockout mice. Physiol Rep 4: 4, 2016.

23. Wang GQ, Tang T, Wang ZS, Liu YY, Wang L, Luo PF and Xia ZF: Overexpression of hypo-phosphorylated iкB $\beta$ at Ser313 protects the heart against sepsis. PLoS One 11: e0160860, 2016.

24. Kenneth J: Livak, Thomas D. Schmittgen; Analysis of relative gene expression data using real-time quantitative PCR and the $2^{-\Delta \Delta \mathrm{CT}}$ method. Methods 25: 402-408, 2001.

25. Iskander KN, Osuchowski MF, Stearns-Kurosawa DJ, Kurosawa S, Stepien D, Valentine C and Remick DG: Sepsis: Multiple abnormalities, heterogeneous responses, and evolving understanding. Physiol Rev 93: 1247-1288, 2013.

26. LilleyE,Armstrong R,ClarkN, Gray P,Hawkins P,Mason K,LópezSalesansky N, Stark AK, Jackson SK, Thiemermann C, et al: Refinement of animal models of sepsis and septic shock. Shock 43: 304-316, 2015.

27. Kuzmich NN, Sivak KV, Chubarev VN, Porozov YB, Savateeva-Lyubimova TN and Peri F: TLR4 signaling pathway modulators as potential therapeutics in inflammation and sepsis. Vaccines (Basel) 5: 5, 2017.

28. Beutler B, Du X and Poltorak A: Identification of Toll-like receptor 4 (Tlr4) as the sole conduit for LPS signal transduction: Genetic and evolutionary studies. J Endotoxin Res 7: 277-280, 2001.

29. Płóciennikowska A, Hromada-Judycka A, Borzęcka K and Kwiatkowska K: Co-operation of TLR4 and raft proteins in LPS-induced pro-inflammatory signaling. Cell Mol Life Sci 72: 557-581, 2015.

30. Li P, Chen XR, Xu F, Liu C, Li C, Liu H, Wang H, Sun W, Sheng YH and Kong XQ: Alamandine attenuates sepsis-associated cardiac dysfunction via inhibiting MAPKs signaling pathways. Life Sci 206: 106-116, 2018.

31. Jeong HS, Lee TH, Bang CH, Kim JH and Hong SJ: Risk factors and outcomes of sepsis-induced myocardial dysfunction and stress-induced cardiomyopathy in sepsis or septic shock: A comparative retrospective study. Medicine (Baltimore) 97: e0263, 2018.

32. Sevilla Berrios RA, O'Horo JC, Velagapudi V and Pulido JN: Correlation of left ventricular systolic dysfunction determined by low ejection fraction and 30-day mortality in patients with severe sepsis and septic shock: A systematic review and meta-analysis. J Crit Care 29: 495-499, 2014.

33. Haileselassie B, Su E, Pozios I, Niño DF, Liu H, Lu DY, Ventoulis I, Fulton WB, Sodhi CP, Hackam D, et al: Myocardial oxidative stress correlates with left ventricular dysfunction on strain echocardiography in a rodent model of sepsis. Intensive Care Med Exp 5: 21, 2017.

34. Vallabhajosyula S, Pruthi S, Shah S, Wiley BM, Mankad SV and Jentzer JC: Basic and advanced echocardiographic evaluation of myocardial dysfunction in sepsis and septic shock. Anaesth Intensive Care 46: 13-24, 2018 
35. Hoffman M, Kyriazis ID, Lucchese AM, de Lucia C Piedepalumbo M, Bauer M, Schulze PC, Bonios MJ, Koch WJ and Drosatos K: Myocardial strain and cardiac output are preferable measurements for cardiac dysfunction and can predict mortality in septic mice. J Am Heart Assoc 8: e012260, 2019.

36. Takashima K, Matsunaga N, Yoshimatsu M, Hazeki K, Kaisho T, Uekata M, Hazeki O, Akira S, Iizawa Y and Ii M: Analysis of binding site for the novel small-molecule TLR4 signal transduction inhibitor TAK-242 and its therapeutic effect on mouse sepsis model. Br J Pharmacol 157: 1250-1262, 2009.

37. Sanfilippo F, Corredor C, Fletcher N, Landesberg G, Benedetto U, Foex P and Cecconi M: Diastolic dysfunction and mortality in septic patients: A systematic review and meta-analysis. Intensive Care Med 41: 1004-1013, 2015.

38. Ran S, Bhattarai N, Patel R and Volk-Draper L: TLR4-induced inflammation is a key promoter of tumor growth, vascularization, and metastasis. In: Translational Studies on Inflammation. 2020.

39. Lin Y, Xu Y and Zhang Z: Sepsis-induced myocardial dysfunction (SIMD): The pathophysiological mechanisms and therapeutic strategies targeting mitochondria. Inflammation 43: 1184-1200, 2020.

40. Fan TT, Feng XY, Yang YZ, Gao F and Liu Q: Downregulation of PI3K- $\gamma$ in a mouse model of sepsis-induced myocardial dysfunction. Cytokine 96: 208-216, 2017.

41. Cheng H, Fan WZ, Wang SC, Liu ZH, Zang HL, Wang LZ, Liu HJ, Shen XH and Liang SQ: N-terminal pro-brain natriuretic peptide and cardiac troponin I for the prognostic utility in elderly patients with severe sepsis or septic shock in intensive care unit: A retrospective study. J Crit Care 30: 654.e9-654.e14, 2015.

42. Klouche K, Pommet S, Amigues L, Bargnoux AS, Dupuy AM Machado S, Serveaux-Delous M, Morena M, Jonquet O and Cristol JP: Plasma brain natriuretic peptide and troponin levels in severe sepsis and septic shock: Relationships with systolic myocardial dysfunction and intensive care unit mortality. J Intensive Care Med 29: 229-237, 2014.

43. Zhang T, Yan T, Du J, Wang S and Yang H: Apigenin attenuates heart injury in lipopolysaccharide-induced endotoxemic model by suppressing sphingosine kinase 1/sphingosine 1-phosphate signaling pathway. Chem Biol Interact 233: 46-55, 2015.
44. Yu X, Jia B, Wang F, Lv X, Peng X, Wang Y, Li H, Wang Y, Lu D and Wang $H: \alpha_{1}$ adrenoceptor activation by norepinephrine inhibits LPS-induced cardiomyocyte $\mathrm{TNF}-\alpha$ production via modulating ERK1/2 and NF- $\kappa$ B pathway. J Cell Mol Med 18: 263-273, 2014.

45. Wang Z, Bu L, Yang P, Feng S and Xu F: Alleviation of sepsis induced cardiac dysfunction by overexpression of Sestrin 2 is associated with inhibition of $\mathrm{p}$ S6K and activation of the pAMPK pathway. Mol Med Rep 20: 2511-2518, 2019.

46. Suzuki T, Suzuki Y, Okuda J, Kurazumi T, Suhara T, Ueda T, Nagata $\mathrm{H}$ and Morisaki $\mathrm{H}$ : Sepsis-induced cardiac dysfunction and $\beta$-adrenergic blockade therapy for sepsis. J Intensive Care 5: 22, 2017.

47. Zhang X, Li M, Wang H and Astragaloside IV: Astragaloside IV alleviates the myocardial damage induced by lipopolysaccharide via the Toll-like receptor 4 (TLR4)/nuclear factor kappa B $(\mathrm{NF}-\kappa \mathrm{B}) /$ proliferator-activated receptor $\alpha(\mathrm{PPAR} \alpha)$ signaling pathway. Med Sci Monit 25: 7158-7168, 2019.

48. Natanson C, Eichenholz PW, Danner RL, Eichacker PQ, Hoffman WD, Kuo GC, Banks SM, MacVittie TJ and Parrillo JE: Endotoxin and tumor necrosis factor challenges in dogs simulate the cardiovascular profile of human septic shock. J Exp Med 169: 823-832, 1989.

49. Cain BS, Meldrum DR, Dinarello CA, Meng X, Joo KS, Banerjee A and Harken AH: Tumor necrosis factor-alpha and interleukin-1beta synergistically depress human myocardial function. Crit Care Med 27: 1309-1318, 1999.

50. Boillot A, Capellier G, Racadot E, Wijdenes J, Herve P and Barale F: Pilot clinical trial of an anti-TNF alpha monoclonal antibody for the treatment of septic shock. Clin Intensive Care 6: 52-56, 1995.

51. Herbertson MJ, Werner HA, Goddard CM, Russell JA, Wheeler A, Coxon R and Walley KR: Anti-tumor necrosis factor-alpha prevents decreased ventricular contractility in endotoxemic pigs. Am J Respir Crit Care Med 152: 480-488, 1995.

This work is licensed under a Creative Commons Attribution-NonCommercial-NoDerivatives 4.0 International (CC BY-NC-ND 4.0) License. 